\title{
Author Correction: Reproducibility: No expiration date
}

Michelle M. Kuttel (D)

Correction to: Nature Astronomy https://doi.org/10.1038/s41550-021-01402-3, published online 21 June 2021.

In the version of this News \& Views article originally published, in the sentence beginning "To help with this..., "Maneage" was misspelled as "Manaege". In addition, in the sentence beginning "Those with a training..., Edsger Dijkstra's first name was misspelled as "Edsgar". All versions of the article have now been amended.

Published online: 18 August 2021

https://doi.org/10.1038/s41550-021-01477-y

๑) Springer Nature Limited 2021 